\title{
Problema de roteirização de veículos com janelas de atendimento, frotas heterogêneas e entregas fracionadas
}

\author{
Marc Antonio Vieira de Queiroz ${ }^{1}$, Pedro Casagrande Campos ${ }^{1}$, \\ Rodolfo Miranda de Barros ${ }^{1}$, Jacques Duílio Brancher ${ }^{1}$ \\ ${ }^{1}$ Departamento de Computação - Universidade Estadual de Londrina (UEL) \\ Caixa Postal 6.001 - 86.051-990 - Londrina - Pr - Brasil \\ marc.queiroz@gmail.com,pccampos@gmail.com, \{rodolfo, jacques\}@uel.br
}

\begin{abstract}
This paper presents a solution to the vehicle routing problem based on the technique of building routes using sequential insertion. The construction of routes is based on savings heuristics which is inspired on classical algorithms, such as developed by Clarke and Wright in 1964. The heuristics employed makes use of four algorithms, Combined Savings (CS), Optimistic Opportunity Savings (OOS), Realistic Opportunity Savings (ROS) and Realistic Opportunity Savings with a route shape parameter $\left(R O S_{\lambda}\right)$. The proposed algorithms are tested by a set of benchmarks and presents graphic and numeric solutions.
\end{abstract}

Resumo. Este artigo apresenta uma solução para o problema de roteirização de veículos utilizando a técnica de construção de rotas por inserção sequencial. A construção de rotas baseia-se na heurística de economia inspirada em algoritmos clássicos, como o desenvolvido por Clarke e Wright em 1964. A heurística de economia empregada faz uso de quatro algoritmos, Economia Combinada (EC), Oportunidade Otimista de Economia (OOE), Oportunidade Real de Economia (ORE) e Oportunidade Real de Economia com parâmetro modelador de rota $\left(O R E_{\lambda}\right)$. Os algoritmos são testados por um conjunto de benchmarks e apresentam soluções gráficas e numéricas.

\section{Introdução}

O crescimento populacional e a ocupação desordenada nas metrópoles têm exigido das empresas de logística a criação de novos centros de distribuição. Este tipo de situação obriga as empresas a adotarem estratégias para minimizar seus custos operacionais na distribuição das encomendas aos clientes em sua periferia. Para isso utilizam-se de técnicas de roteirização de veículos para as operações de entrega.

Com o crescimento da competição e da evolução dos serviços, os clientes finais tem se tornado mais exigentes quando se tratam de horários, o que trouxe um novo desafio à tarefa de distribuição. Ao incluir restrições de tempo na realização de entregas também houve a inclusão de novas necessidades e requisições exigidas por parte do cliente. Por isso a concorrência entre empresas terceirizadas de roteirização tem crescido. E quem oferece o serviço mais adaptado ao cliente acaba ganhando destaque no ramo.

$\mathrm{Na}$ busca por oferecer melhores serviços em um tempo mais curto, passando assim confiabilidade aos clientes, as empresas de transporte têm procurado a saída para os desafios através de sistemas de roteirização de veículos, que objetivam construir e organizar rotas econômicas para distribuição de encomendas a partir de um depósito central. 
Aumentando assim a produtividade e visibilidade dos seus clientes no mercado em que atuam.

O problema abordado trata da roteirização de veículos de entrega com capacidade de transporte mista e que obedeçam a janelas de atendimento, na literatura estes problemas são conhecidos como VRPMVTTWSD (Vehicle Routing Problem with Multiple Vehicle Types, Time Windows and Split Deliveries) [Belfiore and Yoshida Yoshizaki 2009]. Os sistemas de roteirização de veículos (SRV), ou simplesmente roteirizadores, tiveram os primeiros estudos de casos e aplicação em [Semet and Taillard 1993], [Rochat and Semet 1994] e [Brandão 1997].

Nesses casos citados e em estudos mais recentes os resultados da aplicação dos SRV se mostraram satisfatórios, no Brasil um exemplo é a aplicação desta metodologia num grupo varejista que teve seus custos com transportes reduzidos em $8 \%$ [Belfiore and Yoshida Yoshizaki 2009].

Considerando-se o acima exposto, a proposta desse trabalho é apresentar um SRV levando em consideração restrições de janelas de atendimento e uma frota de veículos mista com entregas fracionadas. O sistema tem como base métodos definidos por [Liu and Shen 1999], e por [Dullaert et al. 2002], que aplicam a heurística de inserção sequencial definida inicialmente por [Solomon 1987].

Para este fim, o trabalho está dividido da seguinte forma: Na seção 2, é apresentada a formulação do problema . A seguir, na 3 são apresentadas as heurísticas de economia que foram utilizadas para o desenvolvimento do código computacional. Na seção 4 é apresentada a metodologia utilizada.

\section{Formulação do Problema}

Esta seção define as variáveis que compõem o problema, bem como as heurísticas de economia para o VRPMVT que apresenta similaridades com o problema de janelas de atendimento.

Este trabalho estuda um problema real de roteirização de veículos com frota heterogênea, janelas de atendimento e entregas fracionadas (VRPMVTTWSD ${ }^{1}$ ) na empresa brasileira RoadSolution ${ }^{\circledR}$ prestadora de serviços de logística. A RoadSolution ${ }^{\circledR}$ oferece seus serviços para empresas transportadoras que desejam fazer uso do seu SRV.

O problema é baseado em um único centro de distribuição por transportadora, ou região de atuação, e pode realizar um número variável de entregas. As entregas são distribuídas através de uma frota de veículos heterogêneas. A frota de veículos pode pertencer a transportadora, cliente da RoadSolution, quanto a terceiros contratados.

Cada veículo relacionado a transportadora possuí cadastrado seu custo de aquisição, fixo e variável. O ciclo de distribuição são de 24 horas, onde a demanda é conhecida antecipadamente pela coleção de notas fiscais cadastradas no SRV. Cada cliente pode ser atendido por mais de um veículo, utilizando a definição de entregas fracionadas. Isso pode acontecer quando alguma entrega possuir demanda superior a capacidade do maior veículo da frota disponível.

\footnotetext{
${ }^{1}$ Doravante denominada de VRP
} 
Os clientes podem possuir restrições de janela de tempo e restrições de acessibilidade (ex: alguns clientes não possuem área suficiente para receber grandes caminhões). As hipóteses relacionadas ao centro de distribuição:

- A demanda dos clientes é conhecida;

- Planejamento de entrega de um dia;

- O tempo de entrega é determinado por ponto atendido, portanto variável;

A formulação matemática do modelo apresentado está listada a seguir.

\subsection{Clientes}

O problema é dado por um conjunto de clientes $N=1,2, \cdots, n$, localizados em $n$ posições distintas, o ponto 0 é definido como o depósito central. Cada par de localizações $(i, j)$, onde $i, j \in N$ e $i \neq j$, está associado com uma viagem de tempo $t_{i j}$ e a distância percorrida $d_{i j}$. Os valores de $t_{i j}$ e $d_{i j}$ são simétricos, ou seja, $t_{i j}=t_{j i}$ e $d_{i j}=d_{j i}$. A demanda no ponto $i$ é $q_{i} \operatorname{com} i=1,2, \cdots, n$.

\subsection{Frota de veículos}

Todos os clientes são visitados a partir de um depósito central por uma frota heterogênea, com os veículos deixando e retornando ao depósito central. A demanda de cada cliente é conhecida. Para problemas com frota heterogêneas tem-se um conjunto $T=1, \cdots, k$ de diferentes tipos de veículos.

Um veículo do tipo $k \in T$ tem a capacidade $a_{k}$, um custo fixo $f_{k}$ e um custo variável por unidade de distância $g_{k}$. O custo do veículo do tipo $k \in T$ realizar o percurso $(i, j)$ é denotado por $c_{i j}^{k}$, no qual é obtido pela multiplicação da distância $d_{i} j$ pelo custo $g_{k}$. O número de veículos do tipo $k$ é assumido como ilimitado $\left(n_{k}=\infty, k \in T\right)$.

Existem restrições de acessibilidade com relação ao tipo de caminhão que realiza as entregas. A maioria dos casos estão ligados a questões operacionais como veículos muito grandes. Nestas situações podem-se assumir que o custo $c_{i j}=\infty$ quando nem o cliente $i$ ou $j$ podem ser atendidos pelo veículo do tipo $k$.

Seja $R_{i}=r_{i}(1), \cdots, r_{i}\left(n_{i}\right)$ a rota do veículo $i$, onde $r_{i}(j)$ é o índice do $j$-ésimo cliente visitado e $n_{i}$ é o número de clientes na rota. Assume-se que toda rota termine no ponto central, $r_{i}\left(n_{i}+1\right)=0$.

\subsection{Janela de atendimento}

Cada cliente $i \in\{1,2, \cdots, n\}$ deve ser atendido. Cada cliente possui uma restrição de janela de tempo $\left[a_{i}, b_{i}\right]$ tal que $a_{i} \leq b_{i}$, que corresponde, respectivamente ao horário inicial e final em que pode ser iniciado o atendimento. O tempo de atendimento $s_{i}$ representa $\mathrm{o}$ tempo de descarga dos veículos (tempo médio de todos os processos administrativos e de operação). Uma rota pode ser formada por um único cliente ou por vários clientes.

\subsection{Entregas Fracionadas}

A demanda de cada cliente pode ser atendida por um ou mais veículos. Isso ocorre em casos onde a demanda excede a capacidade do maior veículo disponível para entrega, ou nos casos que o fracionamento se mostrar eficiente em relação ao custo. 
As variáveis de decisão do modelo são:

$$
x_{i j}^{k}= \begin{cases}1, & \text { se } j \text { é atendido após } i \text { pelo veículo do tipo } k \\ 0, & \text { caso contrário. }\end{cases}
$$

$b_{i}^{k}=$ momento de início de atendimento do cliente i pelo veículo do tipo $k, i=$ $1, \cdots, n$ e $v=1, \cdots, T$.

$y_{i}^{k}=$ fração da demanda do cliente que é realizado pelo veículo do tipo $k$.

O objetivo do modelo é minimizar a distância viajada respeitando a restrição da janela de atendimento. Baseado nos trabalhos de [Dror and Trudeau 1989] e [Ho and Haugland 2004], onde o objetivo é encontrar:

$$
\min \sum_{i=0}^{n} \sum_{j=0}^{n} \sum_{k=1}^{m} d_{i j} x_{i j}^{k}
$$

Além desta equação principal que precisa ser resolvida, há uma série de restrições que devem ser observadas. Tais restrições podem ser encontradas de forma detalhada em [Ho and Haugland 2004].

\section{Heurísticas de Economia VRPMVT}

O algoritmo mais famoso, de [Clarke and Wright 1964] (CW), foi desenvolvido para resolver o problema clássico de VRP. Seja $c_{i j}$ o custo de transporte do cliente $i$ para o $j$. Nesse algoritmo, $c_{i j}$ pode ser igual a distância $d_{i j}$ ou $t_{i j}$, no qual cada cliente é inicialmente servido por um único veículo.

Duas rotas distintas contendo os clientes $i$ e $j$ podem ser combinadas se $i$ e $j$ são o primeiro e o último cliente de suas respectivas rotas, e se a demanda total combinada não exceder a capacidade do veículo. [Clarke and Wright 1964] definiu que a economia que pode ser alcançada combinando duas rotas em uma é: $S_{i j}=c_{i, 0}+c_{0, j}-c_{i, j}$. Para cada iteração as duas rotas com a maior economia são unidas. Este procedimento incremental continua até que nenhuma rota possa ser combinada.

Pode ser visto que CW ignora o custo de aquisição do veículo. Então é esperado que a utilização do algoritmo puro de CW em VRPMVT resultará em uma solução deficiente. Consequentemente, [Golden et al. 1984] modificou a fórmula de CW considerando os custos de aquisição de uma maneira explicita. A seguir, serão apresentados uma revisão dos quatro algoritmos de economia propostos no trabalho:

\section{(1) Economia Combinada (EC)}

Seja $F(z)$ o custo de aquisição do menor veículo que pode servir a rota com uma demanda total igual a $z$. Se $k$ é o primeira ou última entrega da rota, utiliza-se $z_{k}$ para expressar a demanda naquela rota. Claramente, duas rotas com demanda total, respectivamente de $z_{i}$ e $z_{j}$ pode ser combinado por um veículo que custa $F\left(z_{i}+z_{j}\right)$. Portanto, a economia combinada das duas rotas é:

$$
S_{i, j}^{1}=S_{i, j}+F\left(z_{i}\right)+F\left(z_{j}\right)-F\left(z_{i}+z_{j}\right)
$$




\section{(2) Oportunidade Otimista de Economia (OOE)}

Como possíveis economias são ignoradas nas combinações futuras, a EC tende a ser falha na combinação de duas rotas. A heurística OOE considera o potencial extra de economia através da capacidade não utilizada, após a união de duas rotas. Seja $P(z)$ a capacidade do menor veículo que pode servir a rota com uma demanda de $z$. [Golden et al. 1984] define a fórmula de OOE como segue:

$$
S_{i, j}^{2}=S_{i, j}^{1}+F\left(P\left(z_{i}+z_{j}\right)-z_{i}-z_{j}\right)
$$

\section{(3) Oportunidade Real de Economia (ORE)}

Até mesmo para uma pequena quantidade de $z$, a fórmula OOE é otimista, pois assume que a capacidade não utilizada de $z$ pode sempre economizar uma rota que tem a demanda menor ou igual a capacidade do veículo $P(z)$. Para evitar a sobre combinação de rotas, a ORE revisa a fórmula de OOE da seguinte forma: Primeiro, a oportunidade de economia será adicionada a fórmula, se a combinação de duas rotas resultar no uso de um veículo maior do que o atual alocado para a rota. Segundo, o valor da oportunidade de economia é o custo de aquisição do maior veículo que tem a capacidade menor ou igual a capacidade não utilizada depois da união das duas rotas. Seja $F^{\prime}(z)$ o custo de aquisição do maior veículo no qual a sua capacidade seja menor ou igual a $z$. Então a fórmula de economia do procedimento ORE pode ser expressa como segue:

$$
\begin{aligned}
& S_{i, j}^{3}=S_{i, j}^{1}+\delta(\tau) F^{\prime}\left(P\left(z_{i}+z_{j}\right)-z_{i}-z_{j}\right), \\
& \text { onde } \\
& \tau=P\left(z_{i}+z_{j}\right)-P\left(\max \left(z_{i}, z_{j}\right)\right), \\
& \delta(\tau)= \begin{cases}0 & \text { se } \tau=0 \\
1 & \text { se } \tau>0\end{cases}
\end{aligned}
$$

(4) ORE com um parâmetro modelador de rota $\lambda\left(O R E_{\lambda}\right)$

$\mathrm{O}$ algoritmo $O R E_{\lambda}$ simplesmente modifica a fórmula de economia do $\mathrm{CW}$ para $c_{0, i}+$ $c_{j, 0}-\lambda c_{i, j}$. Então a nova fórmula de economia para $O R E_{\lambda}$ é dada por:

$$
S_{i, j}^{4}=S_{i, j}^{3}+(1-\lambda) c_{i, j}
$$

Em contraste com as três fórmulas anteriores, o algoritmo $\left(O R E_{\lambda}\right)$ resolve cada problema variando os valores de $\lambda$ a partir de um conjunto pré-definido de valores. $\mathrm{O}$ melhor resultado dessas execuções é selecionado como final.

Os quatro algoritmos acima focam na modificação da fórmula de economia de $\mathrm{CW}$, mesmo assim apresentam similaridades. Outro fator de destaque, uma vez que duas rotas são combinadas, no próximo passo iterativo do algoritmo, será necessário recalcular os valores daquela rota. Com a utilização de técnicas de programação dinâmica é possível melhorar a performance da execução. 


\section{Metodologia para o VRP}

Uma rota pode conter um ou mais clientes. Seja TIPO-I e TIPO-II o conjunto de rotas contendo somente um cliente ou contendo no mínimo dois clientes, respectivamente. Para os algoritmos de economia demonstrados anteriormente, quaisquer duas rotas podem ser combinadas caindo em um dos seguintes 3 casos: (1) TIPO-I, TIPO-I, (2) TIPO-II, TIPO-I e (3) TIPO-II,TIPO-II.

Um exemplo das combinações podem ser acompanhados na figura 1. No entanto pode-se interpretar esses casos através de um ponto de vista de inserção, ao invés do conceito tradicional de combinação. Sabe-se que as rotas construídas para o problema VRPMVTTW são direcionados. Para estender a explicação supõe-se para uma rota direcionada do TIPO-II, seja (0-f-...-g-0), chama-se a orientação (f-...-g) de caminho genérico e (g-...-f) de caminho inverso.
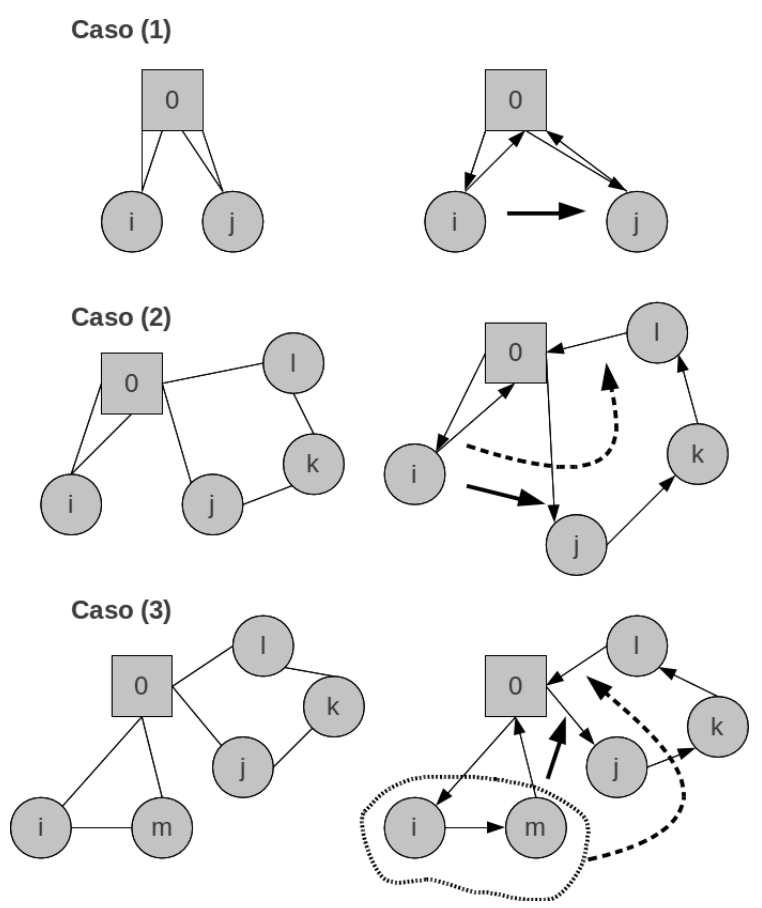

Figura 1. Inclusão de rota pela perspectiva de inserção

Com a inserção pela orientação dos clientes pode-se combinar as operações dos algoritmos baseados em CW. Segundo [Solomon 1987] algoritmos que aplicam inserção incremental geram resultados melhores para solução de problemas com janelas de atendimento. Todos as arestas de uma rota pode gerar uma nova combinação na inserção de um novo ponto de entrega. Portanto a maneira de encontrar um possível resultado é calcular a economia para cada combinação possível na rota. Para lidar com o problema do VRPMVTTW desta forma, é necessário descrever as condições que tornam viáveis a execução das rotas.

\section{Resultados Computacionais e Discussões}

Para testar a performance computacional dos métodos propostos foram utilizados os benchmarks propostos por [Solomon 1987]. As modificações em relação ao benchmark estão 
na utilização de frota de veículos com diferentes capacidades de transporte e uso de entregas fracionadas.

Quanto as entregas fracionadas o sistema RoadSolution ${ }^{\circledR}$ 2, realiza a anotação de pendência para as notas fiscais que apresentam demanda superior a capacidade de transporte do maior veículo. A técnica empregada para o fracionamento das entregas é a mesma utilizada por [Belfiore and Yoshida Yoshizaki 2009]. No pré-processamento das notas fiscais, o fracionamento acontece dividindo-se automaticamente ou manualmente a demanda do cliente, de tal forma a não superar a capacidade máxima de transporte da frota disponível.

Os problemas apresentados podem ser agrupados em seis diferentes categorias: $\mathrm{R} 1, \mathrm{C} 1, \mathrm{RC} 1$ e $\mathrm{R} 2, \mathrm{C} 2, \mathrm{RC} 2$. As letras $\mathrm{R}, \mathrm{C}$ e $\mathrm{RC}$ dizem respeito a forma como os pontos foram gerados. $\mathrm{R}$ para randômico, $\mathrm{C}$ para agrupados do inglês clustered e RC combinação de randômico com agrupados. Os números 1 e 2 dizem respeito as janelas de atendimento, o número 1 são para janelas de atendimento mais curtas e o número 2 para janelas de atendimento mais longas.

Abaixo será apresentada uma solução por grupo, totalizando seis testes. Cada um dos testes irá apresentar a distribuição visual dos clientes (pontos de entrega) e o depósito central. Os clientes são representados por círculos, pontos desconectados são pendentes de entrega. Os depósitos são representados por quadrados. Nos testes realizados os depósitos estão "geocodificados" em dois grupos os R e C, RC. A solução para as rotas é apresentada por arestas ligando os clientes e o depósito, com origem e fim no depósito central, a quantidade de veículos utilizados também define o número de rotas para a solução apresentada.

Os algoritmos utilizados foram o de Economia Combinada(EC), Oportunidade Otimista de Economia(OOE), Oportunidade Real de Economia(ORE) e Oportunidade Real de Economia com parâmetro modeladorORE $E_{\lambda}$. Serão apresentadas as melhores soluções para cada grupo de teste utilizando métricas como distância total percorrida pela frota, taxa de ocupação, número e quantidade de veículos utilizados e custo total do transporte realizado.

Todas as figuras utilizadas para a representação dos grupos de testes foram retiradas do software RoadSolution ${ }^{\circledR}$, segue uma breve introdução ao SRV. A aplicação utiliza uma plataforma cliente-servidor. O servidor foi desenvolvido em Java, utilizando SpringFramework 2.0, banco de dados Postgres-8.4, Postgis-1.5 e Pgrouting. A comunicação com cliente é realizado através de uma aplicação Rich Internet Application(RIA), utilizando Adobe Flex, integrada com o servidor através do BlazeDS (Adobe Remoting Message System). A aplicação Flex utiliza o sistema de visualização de mapas OpenScales para traduzir visualmente as informações geoespaciais.

A versão do SRV utilizada para execução dos benchmarks foi modificada especialmente para testar eficiência dos algoritmos implementados. Portanto o percurso entre dois pontos será a distância euclidiana e o tempo de viagem será igual a essa distância. Na versão do software em produção os percursos são baseados em velocidades médias retiradas dos mapas digitais da malha viária brasileira. O resultado visual desse percurso no SRV é muito parecido com a rota entre dois endereços gerado pelo GoogleMaps ${ }^{\circledR}$, aplicativo geoespacial do Google ${ }^{\circledR}$ disponível na internet. A diferença é que a implementação 
do SRV utiliza uma pilha de softwares independentes e de código aberto.

A tabela 1 mostra a configuração dos veículos utilizados, e a tabela 2 apresenta um resumo dos 6 problemas que foram executados com o software RoadSolution ${ }^{\circledR}$.

Tabela 1. Tipos de veículos utilizados nos testes.

\begin{tabular}{|c|c|c|c|}
\hline Veículo & Capacidade & Custo Fixo & Custo Variável \\
\hline A & 30 & 50 & 10 \\
\hline B & 50 & 80 & 16 \\
\hline C & 80 & 140 & 26 \\
\hline D & 120 & 250 & 43 \\
\hline E & 200 & 500 & 68 \\
\hline
\end{tabular}

Tabela 2. Resumo das soluções encontradas para os benchmaks testados

\begin{tabular}{|c|c|c|c|c|c|}
\hline Teste & De (UP) & Di (UD) & O.M. (\%) & Veículos & Unidades Monetárias \\
\hline R101 & 1458 & 2538.87 & 81.45 & 42 & 36925.18 \\
\hline C101 & 1810 & 2641.09 & 92.35 & 38 & 49186.48 \\
\hline RC101 & 1724 & 3006.69 & 93.19 & 37 & 51597.44 \\
\hline R201 & 1458 & 2104.63 & 81.91 & 33 & 42443.68 \\
\hline C201 & 1810 & 1578.84 & 89.60 & 17 & 75620.71 \\
\hline RC201 & 1724 & 2309.44 & 91.22 & 26 & 62275.73 \\
\hline
\end{tabular}

\subsection{Testes}

Tabela 3. Legenda para abreviaturas da tabela 2

Sigla significado

De Demanda

Di Distância

O.M. Ocupação média

UP Unidades de peso

UD Unidades de distância 


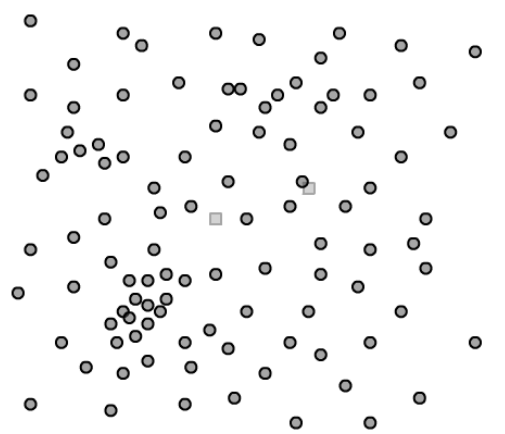

(a) Pendências

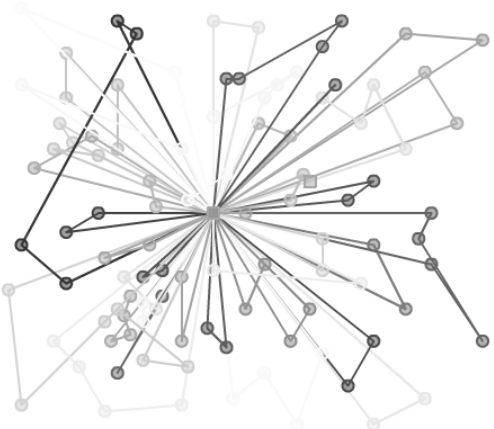

(b) Rotas Geradas

Figura 2. Teste R101

É possível notar na figura $2 \mathrm{a}$ a distribuição randômica de clientes e uma aglomeração na parte inferior esquerda da mesma. A figura $2 b$ apresenta a melhor solução encontrada para R101 que utilizou os algoritmos OOE e EC.

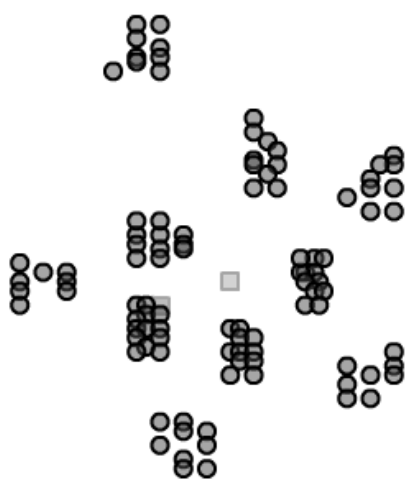

(a) Pendências

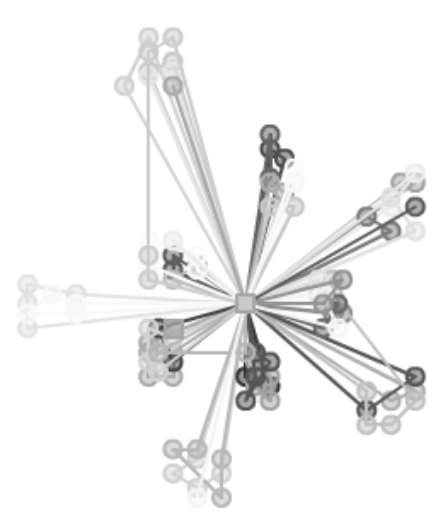

(b) Rotas Geradas

Figura 3. Teste C101

É possível notar na figura 3a a distribuição agrupada dos pontos, apesar disto, o que importa são os requisitos das janelas de atendimento apresentadas por cada cliente. A figura 3b apresenta uma solução encontrada para C101 que utilizou o algoritmo OOE e a estratégia de primeiro ponto mais distante, ou seja, montar as rotas com o ponto inicial mais distante do depósito.

É possível notar na figura 4a a distribuição agrupada dos pontos na periferia da mesma, enquanto a área central apresenta a maioria dos pontos randômicos. A figura 4b apresenta uma solução encontrada para RC101 que utilizou o algoritmo ORE e a estratégia de primeiro ponto mais distante.

A figura 5a apresenta os clientes a serem atendidos, com uma demanda total de 1458 unidades de peso a serem transportadas. Nesse teste as janelas de atendimento são mais alongadas, permitindo maior oportunidade na elaboração de rotas. A figura 5b apresenta a melhor solução encontrada para R201 que utilizou o algoritmo OOE. 


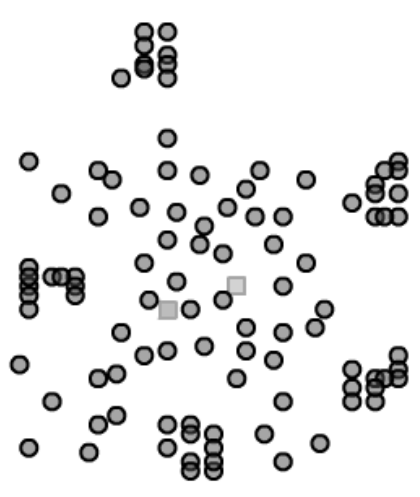

(a) Pendências

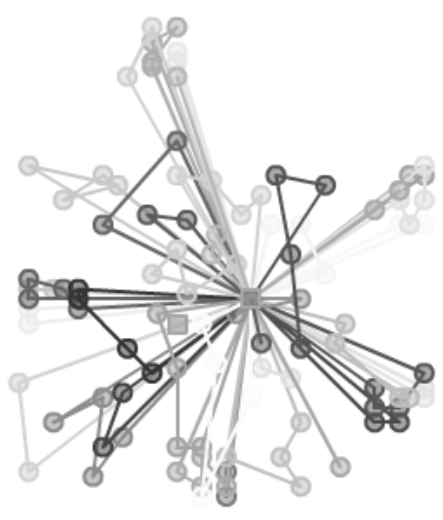

(b) Rotas Geradas

Figura 4. Teste RC101

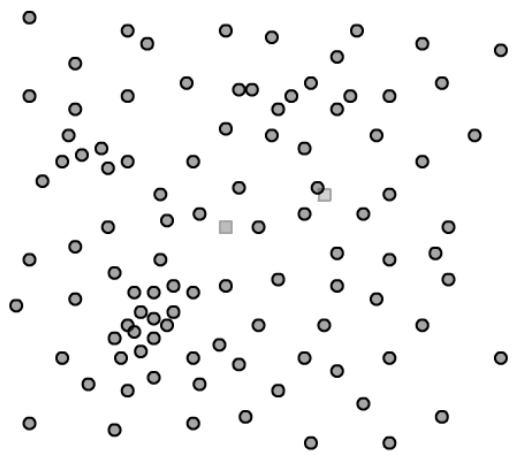

(a) Pendências

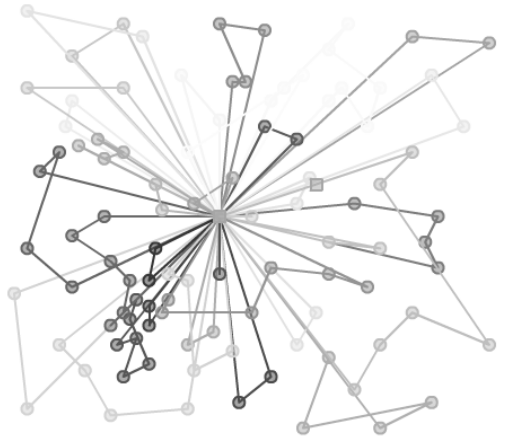

(b) Rotas Geradas

Figura 5. Teste R201

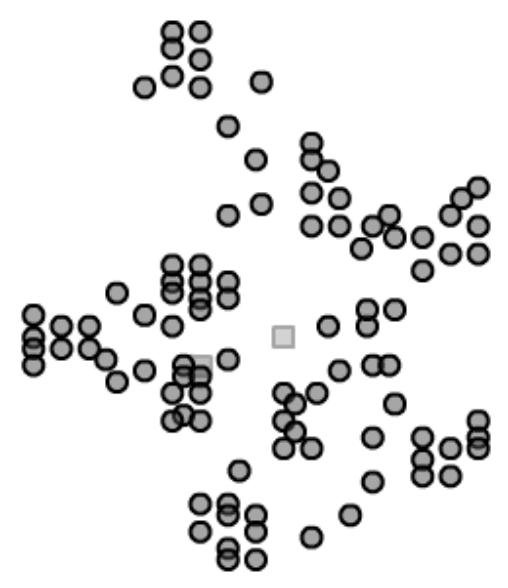

(a) Pendências

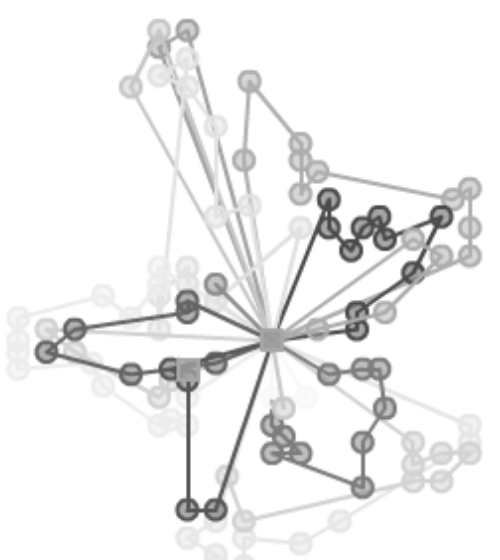

(b) Rotas Geradas

Figura 6. Teste C201 
A figura 6a apresenta os clientes a serem atendidos, com uma demanda total de 1810 unidades de peso a serem transportadas. A figura $6 \mathrm{~b}$ apresenta uma solução encontrada para o grupo de teste C201 que utilizou o algoritmo ORE e a estratégia de primeiro ponto mais próximo.

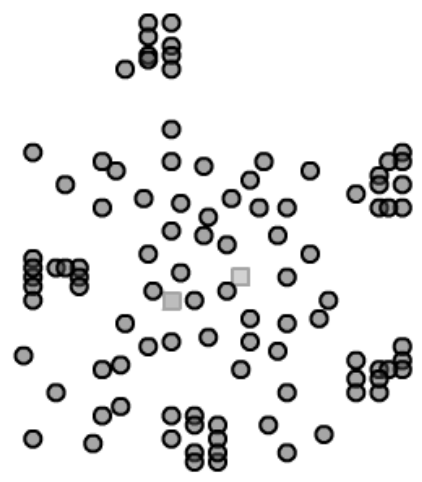

(a) Pendências

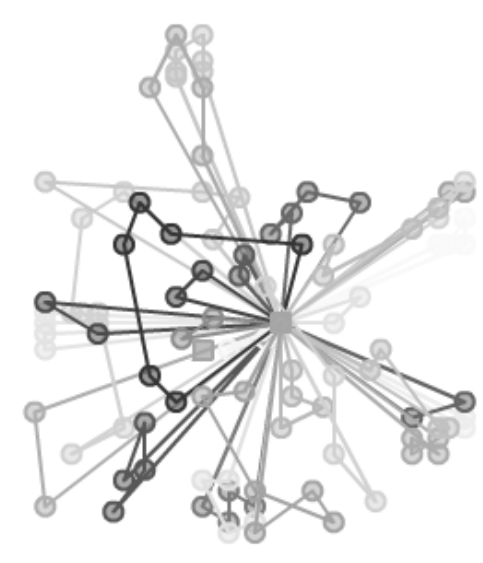

(b) Rotas Geradas

Figura 7. Teste RC201

A figura 7a apresenta os clientes a serem atendidos, com uma demanda total de 1724 unidades de peso a serem transportadas. É possível notar a distribuição agrupada dos pontos na periferia da figura, enquanto a área central apresenta a maioria dos pontos randômicos. A figura $7 \mathrm{~b}$ apresenta uma solução encontrada para RC201 que utilizou o algoritmo OOE e a estratégia de primeiro ponto mais distante.

\section{Conclusão}

Com o objetivo de resolver os problemas de roteirização com frotas heterogêneas, entregas fracionadas e janelas de atendimento, apresentaram-se formulações matemáticas e uma heurística construtiva para atender ao VRP proposto no trabalho.

Os algoritmos apresentados tiveram seus resultados escolhidos entre os fatores, custo do transporte, distância percorrida e taxa de ocupação média dos veículos. A heurística de inserção sequencial não é um método exato de solução, mas sim uma aproximação. Portanto, esse estudo procurou avaliar a eficiência entre os algoritmos quando todos esses fatores são levados em consideração.

Notou-se que o pior resultado é apresentado para os pontos aleatórios. O melhor resultado foi obtido para os pontos agrupados, apresentando uma melhor eficiência para as janelas de atendimento alongadas. No dia-a-dia do software RoadSolution ${ }^{\circledR}$ podese notar que as transportadoras que fazem o uso do SRV definem 3 tipos de janela de atendimento, manhã, tarde e noite. Se as janelas de atendimento do benchmark fossem geradas tendo como escolha um grupo limitado de períodos de entrega as rotas geradas apresentariam uma maior eficiência.

O tempo computacional gasto para execução dos algoritmos é praticamente desprezível, pois utilizou-se percursos euclidianos. Nesse sentido nenhuma referência é feita ao tempo computacional. 
Quanto a utilização dos veículos o algoritmo mostrou-se eficiente pela alta taxa de ocupação alcançada. Esse é um dos fatores comerciais mais importantes para esse tipo de software.

Na revisão bibliográfica realizada não foi possível observar menções a softwares que mesclassem soluções numéricas e visuais para os benchmarks apresentados por [Solomon 1987]. O diferencial das soluções apresentadas é o padrão geométrico das soluções propostas, pois além do resultado numérico é possível acompanhar detalhes do agrupamento das rotas em setores. Ressaltando que o algoritmo de inserção sequencial não emprega nenhum tipo de organização por varredura ou setorização.

A utilização de diferentes algoritmos mostrou que EC e OOE apresentam melhores resultados para encontrar caminhos mais curtos, utilizando veículos de maior capacidade de transporte. E o algoritmo ORE encontra rotas com custo total de transporte mínimo.

\section{Referências}

Belfiore, P. and Yoshida Yoshizaki, H. T. (2009). Scatter search for a real-life heterogeneous fleet vehicle routing problem with time windows and split deliveries in Brazil. European Journal Of Operational Research, 199(3):750-758.

Brandão, J. (1997). A tabu search algorithm for the multi-trip vehicle routing and scheduling problem. European Journal Of Operational Research, 100(1):180-191.

Clarke, G. and Wright, J. W. (1964). Scheduling of Vehicles from a Central Depot to a Number of Delivery Points. Operations Research, 12(4):568-581.

Dror, M. and Trudeau, P. (1989). Savings by Split Delivery Routing. Transportation Science, 23(2):141-145.

Dullaert, W., Janssens, G. K., Sörensen, K., and Vernimmen, B. (2002). New heuristics for the Fleet Size and Mix Vehicle Routing Problem with Time Windows. Journal of the Operational Research Society, 53(11):1232-1238.

Golden, B., Assad, A., Levy, L., and Gheysens, F. (1984). The fleet size and mix vehicle routing problem. Computers \& Operations Research, 11(1):49-66.

Ho, S. C. and Haugland, D. (2004). A tabu search heuristic for the vehicle routing problem with time windows and split deliveries. Computers \& Operations Research, 31(12):1947-1964.

Liu, F.-h. and Shen, S.-y. (1999). A method for vehicle routing problems with multiple vehicle types and time windows. In Proceedings of Natural Science Council.

Rochat, Y. and Semet, F. (1994). A Tabu Search Approach for Delivering Pet Food and Flour in Switzerland. The Journal of the Operational Research Society, 45(11):1233.

Semet, F. and Taillard, E. (1993). Solving real-life vehicle routing problems efficiently using tabu search. Annals of Operations Research, 41(4):469-488.

Solomon, M. M. (1987). Algorithms for the Vehicle Routing and Scheduling Problems with Time Window Constraints. Operations Research, 35(2):254-265. 